\section{$\underset{\substack{\text { hommes } \\ \text { \& migrations }}}{ }$}

\section{Hommes \& migrations}

Revue française de référence sur les dynamiques

migratoires

1307 | 2014

L'Afrique qualifiée dans la mondialisation

\title{
Juifs et Kurdes du Kurdistan : le récit de deux nations
}

\section{Ceng Sagnic}

\section{(2) OpenEdition \\ Journals}

\section{Édition électronique}

URL : http://journals.openedition.org/hommesmigrations/2904

DOI : 10.4000/hommesmigrations.2904

ISSN : 2262-3353

\section{Éditeur}

Musée national de l'histoire de l'immigration

\section{Édition imprimée}

Date de publication : 1 juillet 2014

Pagination : 150-154

ISBN : 978-2-919040-28-5

ISSN : $1142-852 X$

\section{Référence électronique}

Ceng Sagnic, "Juifs et Kurdes du Kurdistan : le récit de deux nations », Hommes \& migrations [En ligne], 1307 | 2014, mis en ligne le 15 janvier 2015, consulté le 22 septembre 2020. URL : http:// journals.openedition.org/hommesmigrations/2904; DOI : https://doi.org/10.4000/ hommesmigrations.2904 


\section{REPÉRAGE}

\section{JUIFS ET KURDES DU KURDISTAN LE RÉCIT DE DEUX NATIONS}

CENG SAGNIC, chargé de recherche au Centre Moshe-Dayan pour les études sur le Moyen-Orient et l'Afrique, Tel-Aviv, Israël. Doctorant à l'université de Tel-Aviv (Israël et de Paris Ouest-Nanterre-La Défense.

es relations judéo-kurdes ont fait l'objet de _nombreuses études malgré la gageure que représente la situation particulière du Kurdistan. Territoire coincé entre quatre pays du MoyenOrient, le Kurdistan est resté longtemps terra incognita pour les historiens jusqu'au regain d'intérêt récent des chercheurs autour de la question des juifs kurdes. Cela depuis la montée en puissance du sionisme et du nationalisme kurde, concrétisés à la fois par la création de l'État d'Israël en 1948 et par les accomplissements kurdes dans l'Irak d'après-guerre.

Selon les données collectées par Israël, environ 25000 juifs kurdes d'Irak ont migré en Israël en 1951-1952. La majorité des juifs kurdes provenaient du Kurdistan irakien, des régions de Mossoul, Dohuk, Akre, Amadiyah, Zebar, Erbil et Kirkouk, même si une importante population juive vivait dans d'autres parties du Kurdistan. Les villes d'Urfa, de Nusaybin et de Diyarbakir du Kurdistan turc, d'Al-Oamishli en Syrie, ainsi qu'une large partie du Kurdistan iranien (Sanandaj, Mahabad et Kermanshah), étaient peuplées de minorités juives jusqu'à la création d'Israël.

Diverses sources avancent les chiffres de 150000 à 200000 juifs kurdes vivant en Israël de nos jours, la plupart dans les environs de Jérusalem. On dit que Mevasseret Zion, une grande ville à proxi- mité de Jérusalem, a été fondée exclusivement par des juifs kurdes. La ville kurdo-irakienne de Zakho était autrefois qualifiée de "Jérusalem du Kurdistan", tandis qu'après l'émigration, c'est le quartier de Mahane Yehuda à Jérusalem qui fut appelé familièrement "petit Kurdistan".

Les traditions kurdes sont toujours observées en Israël, comme par exemple Norouz, le Nouvel An kurde.

\section{Les juifs kurdes à l'époque prémoderne}

Des traces de l'existence de vastes implantations juives dans le Kurdistan sont présentes dans les traditions orales kurde et juive ainsi que dans les itinéraires de voyageurs juifs. Toutefois, les historiens arabes et les écrits des centres talmudiques babyloniens font peu état des juifs du Kurdistan. Il est surprenant que la seule ville judéo-kurde mentionnée dans le Talmud soit Erbil, aujourd'hui capitale de la région du Kurdistan irakien, bien que le Kurdistan ne se trouve qu'à quelques centaines de kilomètres de ces centres de savoir'. Cela témoigne de l'isolement du Kurdistan du reste de la région, avec pour corollaire la relation particulière entre Kurdes et juifs. 
Femmes kurdes en Israël.

(c) Amanda Harding

La Bible mentionne la déportation des Israélites au Kurdistan : "Le roi d'Assyrie prit Samarie, et emmena Israël captif en Assyrie. II les fit habiter à Chalach, et sur le Chabor, fleuve de Gozan, et dans les villes des Mèdes" (2 Rois 17 : 6).

Les écrits de deux voyageurs juifs émérites ayant parcouru le Kurdistan aux XII et XIII e siècles demeurent les uniques sources matérielles renseignant sur la vie sociale et politique des juifs kurdes au début de l'époque prémoderne. Benjamin de Tudèle, qui a voyagé au Kurdistan au début du XII siècle, et Petachiah de Ratisbonne, qui a suivi ses pas au XIII ${ }^{\text {e }}$ siècle, parlent de communautés juives installées en Perse. Les vingt jours de pérégrination de Benjamin de Tudèle dénotent l'étendue territoriale des tribus juives souveraines disséminées autour du fleuve de Gozan. Selon lui, ces tribus n'étaient soumises à aucune autorité non juive, mais étaient gouvernées par un prince dans leur terre isolée, entourée du désert et du fleuve ${ }^{2}$. Hormis la mention de David Alroy (116o ap. J.-C.), un messie né dans la ville kurde d'Amadiyah qui se rebellera contre le roi de Perse dans le but de libérer les juifs exilés et de les ramener en Israël, les écrits des deux voyageurs donnent la vision d'un Kurdistan prospère et dénué de toute intention sioniste prémoderne. Les deux explorateurs racontent que la population juive kurde des deux villes centrales du Kurdistan, à savoir Mossoul et Amadiyah, s'élevait à environ 30 ooo personnes à l'époque. Il semble également que le peuple juif du Kurdistan jouissait d'une autonomie importante à Mossoul, devenue par ailleurs destination de l'immigration juive en provenance de la Syrie-Palestine et de Babylone sous la menace de l'arrivée des croisés ${ }^{3}$. Toutefois, cette prospérité évoquée par Benjamin et Petachiah allait s'étioler durant les deux siècles suivants, suite à l'invasion mongole au XIVe siècle provoquant la destruction de plusieurs villes du Kurdistan. Les Kurdes et les juifs auraient fui aussi loin que l'Égypte et l'Algérie pour échapper aux conquérants mongols. La région connut alors une nouvelle période sombre, d'où l'absence quasi totale de documents sources sur les relations judéo-kurdes des XIV et XVe siècles. La stabilité ne fut retrouvée qu'en 1534, avec la victoire des Ottomans sur l'Empire perse. Cette date marque également le début des rébellions tribales à l'encontre des gouvernements centralisés, qui devaient durer jusqu'à la fin du $X X^{e}$ siècle ${ }^{4}$.

Des manuscrits et documents écrits par des juifs kurdes font leur apparition au XVI siècle sous le règne ottoman. Outre le fait qu'ils constituent la première production écrite par les juifs kurdes eux-mêmes, ces manuscrits renseignent sur leur vie politique et sociale. Parallèlement, les écrits d'un voyageur juif yéménite, Zecha- 


\section{REPÉRAGE}

riah Al-Zahiri, fournissent des détails sur d'autres implantations juives ne figurant pas dans ceux de Benjamin et Petachiah. Ces nouvelles villes s'étendaient de l'actuelle Urfa en Turquie et Nusaybin, ville frontalière syro-turque, jusqu'à Mossoul, Erbil et Kirkouk en Irak. Ces documents, datés des $X V l^{e}$ et $X V I I^{e}$ siècles, témoignent une nouvelle fois de la prospérité des villes juives du Kurdistan. La plupart ont été rédigés par les rabbins d'une famille éminente, les Barzani, dont les descendants sont aussi connus que leurs ancêtres, à la fois en Israël et au Kurdistan irakien. À cette époque, les yechivot (centres d'apprentissage juifs) fondés par la famille Barzani et situés dans les villes de Nerwa et Amadiyah attiraient des étudiants juifs d'Égypte et de la terre d'Israël. Les mêmes sources expliquent que l'une des plus grandes bibliothèques de la région futfondée par le rabbin Natha-

La symbiose judéo-kurde a donné naissance au Kurdistan à une entité civilisationnelle, comme en témoignent

les traditions orales kurde

et juive qui reflètent

leur appréciation mutuelle. nel Barzani, léguée à son fils le rabbin Samuel puis à sa petite-fille, Asenath Barzani, femme rabbin. Première et unique femme rabbin du Kurdistan, AsenathBarzanirepritlachaire d'études religieuses précédemment occupée par son père, qui n'avait pas eu de fils. La légende raconte qu'en plus d'avoir reçu de son père un enseignement biblique, Asenath fut également initiée aux secrets de la Kabbale, courant mystique du judaïsme.

Aux $X V l^{e}$ et $X V \mid I^{e}$ siècles, les rabbins du Kurdistan, principalement de la famille Barzani, en plus de leurs fonctions, dirigeaient également des centres d'études jusque dans les grandes villes de Mossoul et Bagdad 5 .
Juifs célébrant Newroz (C) ARchives

\section{Juifs et Kurdistan à l'époque moderne}

La symbiose judéo-kurde a donné naissance au Kurdistan à une entité civilisationnelle, comme en témoignent les traditions orales kurde et juive qui reflètent leur appréciation mutuelle. Pour beaucoup d'historiens, les juifs du Kurdistan entretenaient de meilleures relations avec leurs voisins non juifs que les juifs d'Europe ${ }^{6}$. Le même constat vaut pour d'autres communautés juives installées non loin du Kurdistan. Dans une interview conduite par Geoffrey Khan, linguiste de renom, un juif de Sina du Kurdistan iranien décrit les relations judéo-kurdes: "Il existe deux types de musulmans, des chiites et des sunnites. Ceux du Kurdistan, en tant que Kurdes, sont sunnites. Ils se sont montrés très bons envers les juifs. Mais les chiites de Hamadn, Téhéran, Ispahan, Chiraz, Kirmân, Khorasan, Baloutchistan, étaient tellement hostiles [envers les juifs] que Dieu a dit 'Ca suffit !'... La situation est tout autre dans notre ville [Sanandaj]. Ils étaient les bienvenus chez nous et à notre table et aimaient y venir." Les juifs du Kurdistan étaient soumis à différentes régle- 
mentations sociales selon qu'ils habitaient en zone urbaine ou rurale. En théorie, les juifs des centres urbains dépendaient uniquement des institutions étatiques, à l'inverse des juifs en zone rurale qui eux étaient soumis à l'autorité de chefs tribaux non juifs. Toutefois, en pratique, la communauté juive était astreinte aux deux systèmes de loi, autrement qualifiés de "bigouvernance" par l'historien israélien Mordechai Zaken. Les juifs ruraux faisaient peu appel aux organismes officiels en ville et s'adressaient plutôt au chef régional, ou âghâ du village. Les juifs urbains répondaient quant à eux à la juridiction officielle, puisque l'autorité des chefs tribaux ne s'étendait pas aux villes. De fait, plusieurs facteurs changeants (faible pouvoir central au Kurdistan, rivalités entre chefs tribaux et autorités centrales) soumettaient les juifs du Kurdistan aux deux systèmes. Zaken insiste sur ce double système de gouvernance: "En pratique, il n'y avait ni gouvernement, ni police dans les régions tribales et le âghâ représentait l'unique autorité pour les juifs. Il était à la fois le gouvernement, la police et la cour tout en un. Son influence sur eux était donc décisive. Au village, les relations entre Kurdes musulmans et juifs étaient dictées essentiellement par l'attitude du chef (âghâ) envers les juifs locaux. En ville, le statut des juifs n'était pas le fait d'une seule personne mais de plusieurs facteurs concomitants comme le niveau de contrôle du gouvernement central, les compétences et le poids financier de la classe dirigeante juive et ses alliances avec l'aristocratie tribaleg." Le statut relativement inférieur des juifs kurdes comparé à celui des musulmans donna lieu à un statu quo défini par Zaken sous le patronage des chefferies tribales kurdes. Si cette situation eut tendance à s'estomper par la suite avec l'avènement d'autorités étatiques plus puissantes, les juifs kurdes demeurèrent les sujets de chefs tribaux kurdes jusqu'à leur évacuation complète en Israël.

Cette façon d'envisager les relations judéo-kurdes sous l'angle du patronage souligne également que les dynamiques sociales de Kurdes et de juifs dépendaient de deux facteurs décisifs. Le premier est l'impact de la géographie isolant le Kurdistan du reste du monde. Jusqu'aux temps modernes et même après, les régions kurdes montagneuses d'Irak, de Turquie et d'Iran rendaient l'exercice d'un pouvoir centralisé extrêmement compliqué. Cela se terminait La plus petite minorité non musulmane du Kurdistan et, pour certains historiens, la vraie représentante des culture et traditions locales, a quitté la région avec ses congénères irakiens pour rejoindre Israël en 1952-1953. tacites entre les chefs tribaux de Kurdes musulmans et leur gouvernement central, au nom de la stabilité politique régionale. Le second facteur important était la lutte kurde pour une indépendance des gouvernements turc, irakien et iranien. Le mouvement nationaliste kurde n'a pas seulement renforcé l'isolement des juifs en tant que minorité vivant au sein d'une autre minorité entourée de vastes armées, il a aussi contraint les juifs kurdes à s'engager dans un sanglant combat sans fin.

Toutefois, les relations entre Kurdes et juifs ne se limitaient pas aux sphères sociale et politique. Tout comme les Kurdes, les juifs kurdes ont développé une riche littérature orale. Yona Sabar explique que la littérature folklorique juive au Kurdistan - comprenant la littérature écrite par des rabbins - trouve ses racines dans la tradition orale kurde. Les écrits et enseignements religieux étaient également transmis par voie de tradition orale, ce qui permit aux générations futures de se familiariser avec la Bible et le judaïsme. Cela pour 


\section{REPÉRAGE}

la partie religieuse de la littérature juive au Kurdistan ; la partie laïque, plus importante, de la littérature dérivait principalement de sources kurdes. L'unique différence avec les contes, poèmes et ballades récités par les juifs kurdes était la langue, puisqu'ils étaient en néo-araméen. La composante laïque du folklore kurde fut donc tout naturellement source d'inspiration pour la tradition orale juive du Kurdistan ${ }^{10}$.

\section{En route pour la Terre promise : la migration des juifs en Israël}

Les motivations du départ massif vers Israël des juifs du Kurdistan furent sans aucun doute différentes de celles des autres communautés juives du Moyen-Orient. Pour autant, le sionisme en tant que concept fondamental du judaïsme n'était pas absent du discours des juifs du Kurdistan. Tout comme pour d'autres communautés juives dans d'autres régions du monde, le mot "Jérusalem" occupait une place centrale dans les prières quotidiennes des juifs kurdes. Toutefois, le concept politique du sionisme ne fut introduit au Kurdistan qu'à la fin de la Seconde
Guerre mondiale, surtout après les pogroms anti-juifs menés par le régime irakien pro-nazi. La peur de l'Holocauste a également contribué à la décision des juifs du Kurdistan d'émigrer en Israël. Un juif kurde témoigne de cette peur : "Durant la Seconde Guerre mondiale d'Hitler, que son nom et sa mémoire restent à jamais effacés, nous avons entendu des rumeurs de massacres de juifs et cela nous a profondément peinés"." La plus petite minorité non musulmane du Kurdistan et, pour certains historiens, la vraie représentante des culture et traditions locales, a quitté la région avec ses congénères irakiens pour rejoindre Israël en 1952-1953. Néanmoins, comme ce fut le cas pour l'histoire juive au Kurdistan, le départ des juifs kurdes s'est fait dans des conditions radicalement différentes de celui des juifs du MoyenOrient et d'Europe. Selon les descendants de ces juifs kurdes présents en Israël, le Kurdistan est l'unique endroit au monde où les juifs sont sortis dans les rues célébrer le retour en terre d'Israël après 2700 ans, tandis que leurs voisins non juifs pleuraient leur départ. Beaucoup prétendent que cette migration massive des juifs kurdes n'a pas mis fin à la symbiose judéo-kurde mais qu'au contraire elle l'a élevée à un tout autre niveau. 